\title{
PANCHARATNAM AND BERRY PHASES IN THREE-LEVEL PHOTONIC SYSTEMS
}

\author{
Y. Ben-Aryeh \\ Physics Department, Technion-Israel Institute of Technology \\ Haifa 32000, Israel \\ e-mail: phr65yb@physics.technion.ac.il; \\ Fax: 972-4-8221514

\begin{abstract}
A theoretical analysis of Pancharatnam and Berry phases is made for biphoton three-level systems, which are produced via frequency degenerate co-linear spontaneous parametric down conversion (SPDC). The general theory of Pancharatnam phases is discussed with a special emphasis on geodesic 'curves'in Hilbert space. Explicit expressions for Pancharatnam, dynamical and geometrical phases are derived for the transformations produced by linear phase-converters. The problem of gauge invariance is treated along all the article.
\end{abstract}




\section{Introduction}

An interesting development in quantum mechanics has been made by Berry [1] who has shown that when a quantum-mechanical state is developing around a closed circuit it obtains a geometrical phase in addition to the well known dynamical phase. Aharonov and Anandan [2] have generalized the use of geometric phase to non-adiabatic time developments. Certain ideas from optics by Pancharatnam [3] have been used for defining also phase changes for partial and non unitary cycles $[4,5]$. The discovery of a topological phase in quantum mechanics has led to a unified approach to various topological phenomena in physics, both at the quantum and classical levels. The connections between the geometric phase and fibre-bundle theories [6-10] have been analyzed. Following Berry's paper many experiments have been developed for measuring the geometric part of the phase change, in a variety of contexts, which are related to Lie group theories. Most observations of Berry's phases are related to SU(2) and Lorentz groups, and there is an enor-

mous amount of literature on such systems. In a previous paper [11] Berry's phases and interference effects for three-level atoms, related to the $\mathrm{SU}(3)$ algebra, have been analyzed, and the comparison between this work and those of other authors have been discussed. In the present article we would like to develop the theory of Pancharatnam and Berry phases for three-level optical systems related to the $\mathrm{SU}(3)$ algebra. More specifically the theory of Pancharatnam phase is developed here for a three-level photonic system which is 
realized by the polarization states of the single mode biphoton field [12-15]. A general review for the unitary evolution of polarized light governed by the $\mathrm{SU}(2)$ group and its relation to topological effects has been published [16]. We extend such treatment to three-level photonic system.

There are close connections between the two concepts of Pancharatnam and Berry phases. Since we are treating an optical system we find it more useful to base our analysis on the concept of Pancharatnam phase, but we would also like to show the connection of our analysis with the concept of Berry phase. For the basic understanding of our analysis for a specific system we find it useful to review shortly the basic theories which are used in our derivations.

The paper is arranged as follows: In Section 2 we show the relation between Berry phase and topology. In Section 3 we describe various properties of the Pancharatnam phase and its relation to the Berry phase. The main part of the paper is given in Section 4, where we analyze possible interference effects which can be obtained in single mode biphoton fields [12-15]. In Section 5 we summarize our results.

\section{Berry Phase Related to Topology}

When a quantum-mechanical (QM) state is evolving adiabatically in time and transported around a circuit as an eigenstate of a Hamiltonian with 
slowly varying parameters, it acquires a geometrical phase factor in addition to the dynamical phase [1]. The geometrical phase factor is given by

$$
\gamma_{n}(T)=i \int_{0}^{T} d t\langle n, \vec{R}(t)|d / d t| n, \vec{R}(t)\rangle=i \oint d \vec{R}(t) \cdot\left\langle n, \vec{R}(t)\left|\vec{\nabla}_{\vec{R}}\right| n, \vec{R}(t)\right\rangle
$$

for a Hermitian Hamiltonian $H(\vec{R}(t))$ with parameters $R_{i}(t)$ which are slowly varying along a closed curve $\mathrm{C}$, in parameter space in time $T$. In the adiabatic approximation, it is assumed that $|n, \vec{R}(t)\rangle$ is an eigenstate of the Hamiltonian:

$$
H(\vec{R}(t))|n, \vec{R}(t)\rangle=E_{n} \vec{R}(t)|n, \vec{R}(t)\rangle
$$

The phase factor acquired by the state $\left|\Psi_{n}(\vec{R}, T)\right\rangle$ consists of two parts:

$$
\left|\Psi_{n}(\vec{R}, T)\right\rangle=\exp \left(i \gamma_{n}(T) \exp \left(-i \int_{0}^{T} E_{n}(\tau) d \tau\right)|n, \vec{R}(t)\rangle\right.
$$

where the first and second exponents on the right side of Eq. (3) represent, respectively, the adiabatic Berry phase and the dynamical phase. The term

$$
\vec{A}_{\vec{R}}(n, t) \equiv i\left\langle n, \vec{R}(t)\left|\vec{\nabla}_{\vec{R}}\right| n, \vec{R}(t)\right\rangle
$$

is referred to as a vector potential. As in electromagnetic theory the vector potential is defined up to a gauge transformation. By performing the transformation

$$
|n, \vec{R}(t)\rangle^{\prime}=\exp \left(i \alpha_{n}(\vec{R})\right\rangle|n, \vec{R}(t)\rangle
$$

we induce a "gauge transformation" on $\vec{A}_{\vec{R}}(n, t)$ :

$$
\vec{A}_{\vec{R}}^{\prime}(n, t)=\vec{A}_{\vec{R}}(n, t)-\vec{\nabla}_{\vec{R}}\left(\alpha_{n}(\vec{R})\right)
$$


¿From the definition of Berry phase given by Eq. (1) it is clear that the Berry phase is gauge invariant for a closed circuit.

Considering the state vector $\left|\Psi_{n}(\vec{R}, t)\right\rangle$, Aharonov and Anandan [2] removed its dynamical phase factor, by defining a new state:

$$
\left|\phi_{n}(\vec{R}, t)=\exp \left[i \int_{0}^{t} h_{n}\left(\vec{R}, t^{\prime}\right) d t^{\prime}\right]\right| \Psi(n, \vec{R}(t)\rangle
$$

where

$$
h_{n}\left(\vec{R}, t^{\prime}\right)=\left\langle\Psi_{n}\left(\vec{R}, t^{\prime}\right) \mid \Psi_{n}\left(\vec{R}, t^{\prime}\right)\right\rangle^{-1} R e\left\langle\Psi_{n}\left(\vec{R}, t^{\prime}\right)\left|H\left(\vec{R}, t^{\prime}\right)\right| \Psi_{n}\left(\vec{R}, t^{\prime}\right)\right\rangle
$$

$\left|\phi_{n}\left(\vec{R}, t^{\prime}\right)\right\rangle$ satisfies the equation:

$$
i d / d t\left|\phi_{n}(\vec{R}, t)\right\rangle=\left[H(t)-h_{n}(\vec{R}, t)\right]\left|\phi_{n}(\vec{R}, t)\right\rangle
$$

Multiplication of this equation on the left by $\left\langle\phi_{n}(\vec{R}, t)\right|$ yields the 'parallel transport' law:

$$
\operatorname{Im}\left\langle\phi_{n}(\vec{R}, t)|d / d t| \phi_{n}(\vec{R}, t)\right\rangle=0
$$

Following fibre-bundle theories [6-10] we refer to Eq. (10) as the 'connection'. The 'total space' $N$, in our analysis, includes all normalized Schrodinger wavefunctions. As is well known it is possible to multiply the wavefunction by a phase factor $\exp (i \theta)$, without changing the physical properties of the quantum state. All wavefunctions which differ only by a phase factor are considered in QM as one ray. The 'base space' $R$, in the present analysis, includes all rays of Schrodinger wavefunctions. There is a projection 
$\pi: N \rightarrow R$ from the total space to its base, i.e. from each wavefunction onto its ray. The 'fibre space' $F$ is related to our analysis by including all wavefunctions which differ only by a phase factor as one 'fibre'.

Let us assume, for simplicity, that an element of the 'bundle' $\left|\phi_{n}(\vec{R}, t)\right\rangle$ can be written

$$
\left|\phi_{n}(\vec{R}, t)\right\rangle=\Omega\left|z_{n}(\vec{R}, t)\right\rangle
$$

where $\left|z_{n}(\vec{R}, t)\right\rangle$ is representative of a certain ray and $\Omega$ is the phase factor, $\Omega=\exp (i \theta)$. On the right side of Eq. (11) $\Omega$ represents the 'vertical' part, which is along the 'fibre', while $\left|Z_{n}(\vec{R}, t)\right\rangle$ represents the 'horizontal' part along the 'base'. Substituting Eq. (11) into the connection, Eq. (10), performing the derivative and integrating this equation over a closed circuit during time $T$ we have two contributions which are equal in magnitude and opposite in sign, so that we obtain:

$$
\operatorname{Arg}(\Omega(T))-\arg (\Omega(0)) \equiv \gamma_{n}(T)=i \oint d \vec{R} \cdot\left\langle\phi_{n}(\vec{R}, t)\left|\vec{\nabla}_{\vec{R}}\right| \phi_{n}(\vec{R}(t)\rangle\right.
$$

The geometrical phase factor is then given in Eq. (12) which is the same relation as that of Eq. (1), but without any adiabatic approximation. One finds according to the above analysis that although the quantum circuit is closed in the 'base space' $\left(\left|z_{n}(T)\right\rangle=\left|z_{n}(0)\right\rangle\right)$ it is open in the 'total space', as $\left|\phi_{n}(T)\right\rangle$ is different from $\left|\phi_{n}(0)\right\rangle$, and this difference is given by the Berry phase. 


\section{Pancharatnam Phase and its Relation to Topological Effects}

\section{a. Definition of Pancharatnam Phase}

Consider two normalized nonorthogonal Hilbert states $|A\rangle$ and $|B\rangle$, and assume further that $|A\rangle$ is exposed to $U(1)$ shift $e^{i \phi}[17]$. The resulting interference pattern is determined by

$$
\left.I=\left|e^{i \phi}\right| A\right\rangle+\left.|B\rangle\right|^{2}=2+2|\langle A \mid B\rangle| \cos [\phi-\arg \langle A \mid B\rangle]
$$

where its maximum is obtained at the Pancharatnam relative phase $\phi_{0} \equiv$ $\arg \langle A \mid B\rangle$. This phase is reduced to the $U(1)$ case whenever $|B\rangle=e^{i \theta}|A\rangle$ as it yields $\arg \langle A \mid B\rangle=\theta$. In the original treatments of the Berry phase $[1,2]$ one considers a quantal system evolving around a closed circuit, from an initial wavefunction $|A\rangle$ to a final wavefunction $|B\rangle$ where $|B\rangle$ is obtained from $|A\rangle$ by a cyclic evolution, i.e., by multiplication with a $U(1)$ phase factor. Although the initial phase of the quantal state (its 'fibre') is defined arbitrarily, the phase difference between the state $|A\rangle$ and $|B\rangle$ is well defined and can be observed by interferometric methods. Pancharatnam provided the physical insight [17] that in a noncyclic evolution if the final wave $|B\rangle$ is superimposed on the initial state $|A\rangle$ only the component $\langle A \mid B\rangle|A\rangle$ along $|A\rangle$ interferes with $|A\rangle$. All other components of $|B\rangle$ which are orthogonal to $|A\rangle$ merely add to the intensity, since their cross terms with $|A\rangle$ vanish. The Pancharatnam 'connection' defines the phase between $|B\rangle$ and $|A\rangle$ as $\phi_{o}=\arg \langle A \mid B\rangle$. The interference amplitude $|\langle A \mid B\rangle|$ differs from unity if the

evolution is non-cyclic $[17,18]$, and this difference leads to reduction of the 
visibility in the interference pattern. The Pancharatnam phase is indeterminate if $|B\rangle$ is orthogonal to $|A\rangle$. Although Pancharatnam phase can be defined also for mixed states [19-21] we restrict the analysis of the present paper to pure states. The use of Berry and Pancharatnam phases to noncyclic evolution raises the problem of gauge invariance which is treated in the following paragraph.

\section{b. Pancharatnam Geometric and Dynamical Phases}

An extensive discussion of the mathematical and physical properties of the Pancharatnam phase has been presented in the articles of Mukanda and Simon [22]. We describe here only a few fundamental properties which are needed for understanding the analysis given in Section 4 for a specific system.

Consider a one dimensional or one parameter 'curve' consisting of a family of wave vectors $|\Psi(s)\rangle$ which is changing continuously as a function of $s . s$ is any parameter by which $\Psi(s)$ is continuously changing (including time as a special case). Assuming that $|\Psi(s)\rangle$ is a unit vector for all $|\Psi(s)\rangle$ we get

$$
\operatorname{Re}\langle\Psi(s) \mid \dot{\Psi}(s)\rangle=0 ; \quad\langle\Psi(s) \mid \dot{\Psi}(s)\rangle=i \operatorname{Im}\langle\Psi(s) \mid \dot{\Psi}(s)\rangle
$$

where the derivatives are according to the parameter $s$. One can change the wavevectors $|\Psi(s)\rangle$ by a "gauge transformation":

$$
|\Psi(s)\rangle \rightarrow\left|\Psi^{\prime}(s)\right\rangle=e^{i \alpha(s)}|\Psi(s)\rangle ; \quad s \epsilon\left[s_{1}, s_{2}\right]
$$


where $\alpha(s)$ is a real smooth function of $s$ in the interval $s \epsilon\left[s_{1}, s_{2}\right]$. For cyclic transformation described in the previous section the Berry phase is independent of the gauge transformation. However, for noncyclic transformation we get

$$
\operatorname{Im}\left\langle\Psi^{\prime}(s) \mid \dot{\Psi}^{\prime}(s)\right\rangle=\operatorname{Im}\langle\Psi(s), \dot{\Psi}(s)\rangle+\dot{\alpha}(s)
$$

¿From the above equations we find that we can construct a functional in Hilbert space which is gauge invariant:

$$
\begin{aligned}
& \arg \left\langle\Psi^{\prime}\left(s_{1}\right), \Psi^{\prime}\left(s_{2}\right)\right\rangle-\operatorname{Im} \int_{s_{1}}^{s_{2}} d s\left\langle\Psi^{\prime}(s) \mid \dot{\Psi}^{\prime}(s)\right\rangle= \\
& \arg \left\langle\Psi\left(s_{1}\right), \Psi\left(s_{2}\right)\right\rangle-\operatorname{Im} \int_{s_{1}}^{s_{2}} d s\langle\Psi(s) \mid \dot{\Psi}(s)\rangle=\text { gauge invariant }
\end{aligned}
$$

This property can be interpreted according to the topological description in the previous section. Here the wavefunction $\Psi(s)\rangle$ includes its 'fibre' i.e. $|\Psi(s)\rangle$ belongs to the total space $N$ where it includes all wavefunctions which differ in a phase factor $\exp (i \theta)$ [changing this phase factor by the gauge transformation is considered in topology as moving along the 'fibre']. On the other hand, the functional appearing in Eq. (17), which is gauge invariant represents a projection from the total space to its basis and is defined as the "geometric phase". We find according to Eq. (17) that by substracting the "dynamical phase", which is defined as $\operatorname{Im} \int_{s_{1}}^{s_{2}} d s\langle\Psi(s) \mid \dot{\Psi}(s)\rangle$, from the Pancharatnam phase, which is defined here as $\arg \left\langle\Psi\left(s_{1}\right), \Psi\left(s_{2}\right)\right\rangle$, we get the geometric phase which is gauge invariant. The interesting point here is that while Berry phase analysis, as given in Section 2, is well defined (gauge invariant) only for cyclic evolution, the geometric phase is well defined also for 
noncyclic evolution (also for mixed states [19-21]). Eq. 17 can be reformulated as [22]:

$$
\begin{aligned}
& \phi_{g}(C)=\phi_{p}(N)-\phi_{\mathrm{dyn}}(N) ; \quad \phi_{p}(N)=\arg \left\{\left\langle\Psi\left(s_{1}\right) \mid \Psi\left(s_{2}\right)\right\rangle\right\} ; \\
& \phi_{\mathrm{dyn}}(N)=\operatorname{Im} \int_{s_{1}}^{s_{2}} d s\langle\Psi(s) \mid \dot{\Psi}(s)\rangle,
\end{aligned}
$$

where $\phi_{p}(N)$ and $\phi_{\text {dyn }}(N)$ are defined over the total space $N$ and each of them is gauge dependent while $\phi_{g}(C)$ is the geometric phase which is defined over the basis space of rays and is gauge invariant. Eqs. (17) and (18) have been restricted to a dependence on only one parameter $s$, but such restriction is valid for the optical system which will be treated in Section 4. For a change of the wavefunction in an interval $\left[s_{1}, s_{2}\right]$ one finds that the Pancharatnam phase is fixed only by the initial and final wavefunctions $\left|\Psi\left(s_{1}\right)\right\rangle$ and $\left|\Psi\left(s_{2}\right)\right\rangle$, respectively. In order to find the geometric phase one should find a way to subtract the dynamical phase which is defined at each point $s$ and is obtained by its integration over the interval $\left[s_{1}, s_{2}\right]$.

One can measure the Pancharatnam phase by applying Eq. (13), but it might be quite difficult to subtract experimentally from it the dynamical phase, although it can be done by using a theoretical calculation. In considering consecutive wavefunctions 'curves' one should take into account that the geometric and Pancharatram phases do not have the additive property [19]. In comparison, the dynamical phase of the total path can be obtained by adding the dynamical phases of the 'curves' from which the total path is composed. Especially interesting are the geodesic 'curves' that extremize 
the distance with respect to a certain metric. In the next paragraph we introduce a metric for the present Hilbert space and show how such extremum can be obtained. The geometric phase which is obtained by subtracting the dynamical phase from the Pancharatnam phase can have global properties while the geodesic 'curve' has also local differential properties. These properties are obtained by extremizing a certain functional, leading to the path with the shortest distance between two points in Hilbert space.

\section{c. Hilbert Space Metric and Geodesics}

The distance between two quantum states $|\Psi(s)\rangle$ and $\left|\Psi\left(s^{\prime}\right)\right\rangle$, representing a quantum system which is developed according to parameter $s$, can be given as $[23,24]$

$$
d L^{2}=1-\left|\left\langle\Psi(s) \mid \Psi\left(s^{\prime}\right)\right\rangle\right|^{2}
$$

As mentioned previously $s$ might represent not only the time $t$ but also any other parameter $s$ by which the wavefunction is continuously developed. The use of Eq. (19) is reasonable since if the ray $|\Psi(s)\rangle$ is orthogonal to the ray $\left|\Psi\left(s^{\prime}\right)\right\rangle$ then the distance between the two states is equal to 1 , while if $\left|\Psi\left(s^{\prime}\right)\right\rangle$ is equal to $|\Psi(s)\rangle$ the distance vanishes. Additional explanation to this definition will be given also later, but let us see first the mathematical derivations which are obtained from this equation.

Assuming two close wavefunctions $|\Psi(s)\rangle$ and $|\Psi(s+d s)\rangle$, then we get:

$$
\langle\Psi(s) \mid \Psi(s+d s)\rangle=1+d s\left\langle\Psi \mid \frac{d}{d s} \Psi\right\rangle+\frac{1}{2} d s^{2}\left\langle\Psi \mid \frac{d^{2}}{d s^{2}} \Psi\right\rangle+0\left(d s^{3}\right)
$$


By using the second derivative of $\langle\Psi(s)| \Psi(s)\langle$ we get

$$
\left\langle\Psi \mid \frac{d^{2}}{d s^{2}} \Psi\right\rangle+\left\langle\frac{d^{2}}{d s^{2}} \Psi \mid \Psi\right\rangle+2\left\langle\frac{d}{d s} \Psi \mid \frac{d}{d s} \Psi\right\rangle=0
$$

By using Eqs. (19-21) one gets, after some algebra [23,24]:

$$
\begin{aligned}
& \left(\frac{d L}{d s}\right)^{2}=\{1-\langle\Psi(s) \mid \Psi(s+d s)\rangle\langle\Psi(s+d s) \mid \Psi(s)\rangle\} / d s^{2} \\
& =\left\langle\frac{d \Psi}{d s} \mid \frac{d \Psi}{d s}\right\rangle-\left\langle\frac{d \Psi}{d s} \mid \Psi\right\rangle\left\langle\Psi \mid \frac{d \Psi}{d s}\right\rangle
\end{aligned}
$$

The derivation of Eq. (22), which has been made by using the definition (19), can be obtained also in a different way. The horizontal component of the tangent vector $d / d s|\Psi(s)\rangle$ is given by $d / d s|\Psi(s)\rangle-\left\langle\Psi(s) \mid \frac{d}{d s} \Psi(s)\right\rangle|\Psi(s)\rangle$. Here we have subtracted from the derivative of the wavefunction its movement along the 'fibre' [25], since it does not change the basis of the wavefunction i.e., its ray. The norm of the above vector is given after a straightforward algebra as:

$$
\left\{\left\langle\frac{d \Psi}{d s}\left|-\left\langle\frac{d}{d s} \Psi \mid \Psi\right\rangle\langle\Psi|\right\}\left\{\frac{d}{d s}|\Psi\rangle-\left\langle\Psi \mid \frac{d}{d s} \Psi\right\rangle|\Psi\rangle\right\}=2\left(\frac{d L}{d s}\right)^{2}\right.\right.
$$

which is equivalent to Eq. (22) up to a multiplication by factor 2, which can be inserted arbitrarily in the definition (19). This equation gives a physical insight for using Eq. (19).

In deriving Eq. (22) one neglects terms which are of order $d s^{3}$ or higher. Given the continuous 'curve' $C$ of the wavefunction $\Psi(s)$ varying continuously as a function of the parameter $s$ from $s_{1}$ to $s_{2}$ one gets the functional $[22,26]$ :

$$
L(C)=\int_{s_{1}}^{s_{2}}\{\langle\dot{\Psi}(s) \mid \dot{\Psi}(s)\rangle-\langle\Psi(s) \mid \dot{\Psi}(s)\rangle\langle\dot{\Psi}(s), \Psi(s)\rangle\}^{1 / 2}
$$


The geodesic curve is obtained by extremizing this functional. Mukanda and Simon [22] have shown that any 'curve' composed of a normalized wavefunction which is changing continuously as a function of $s$ and which obeys the equations:

$$
\begin{gathered}
|\ddot{\Psi}(s)\rangle=-\langle\dot{\Psi}(s) \mid \dot{\Psi}(s)\rangle|\Psi(s)\rangle, \\
\langle\Psi(s) \mid \dot{\Psi}(s)\rangle=0,
\end{gathered}
$$

is a geodesic curve. The path [22, 26-28]

$$
|\Psi(s)\rangle=|A\rangle \cos (s)+\left\{\frac{|B\rangle-|A\rangle\langle A \mid B\rangle}{\sqrt{1-|\langle B \mid A\rangle|^{2}}}\right\} \sin s
$$

where $|A\rangle$ and $|B\rangle$ are the initial and final wavefunctions is a geodesic curve as it fulfills Eqs. (24) and (25).

The use of the geodesic equations (26) for noncyclic $S U(2)$ evolution has been discussed [27]. In a recent interesting article [28] this equation has been applied for discussing possible geometric phase measurement of three-level systems in interferometry. According to Eq. (25) $|\Psi\rangle$ is 'parallel transported' [see Eq. (10)] as it leads to vanishing of the dynamical phase at each point $s$. The 'horizontal' property given by Eq. (25) can be destroyed, but not the geodesic property, by multiplying the function $|\Psi(s)\rangle$ by $e^{i \alpha(s)}$ where $\alpha(s)$ is any continuous function of $s$. Such gauge transformation does not destroy the geodesic property as a geodesic is gauge invariant (any property of the basis of the wavefunctions i.e., of the rays is gauge invariant). 


\section{d. The Vertex Theorem}

Let us assume that we have $N-1$ consecutive wavefunctions 'curves' where the r'th 'curve' is described by the transition $\Psi_{r} \rightarrow \Psi_{r+1}$ and the total path is obtained by summation over $r$ from 1 to $N-1$. Using Eq. (18) we get:

$$
\phi_{g}(C)=\arg \left\langle\Psi_{1} \mid \Psi_{N}\right\rangle-\sum_{r=1}^{N-1} \phi_{\text {dyn }}\left(\Psi_{r} \rightarrow \Psi_{r+1}\right)
$$

Although the Pancharatram and the dynamical phase can be gauge dependent their difference given as $\phi_{g}(C)$ is gauge independent.

For cases in which the geometric phase vanish in all the transitions $\Psi_{r} \rightarrow$ $\Psi_{r+1}$ we get:

$$
\begin{aligned}
& \phi_{g}(C)=\arg \left\langle\Psi_{1} \mid \Psi_{N}\right\rangle-\sum_{r=1}^{N-1} \arg \left\langle\Psi_{r} \mid \Psi_{r+1}\right\rangle= \\
& -\arg \left\{\left\langle\Psi_{N} \mid \Psi_{1}\right\rangle\left\langle\Psi_{1} \mid \Psi_{2}\right\rangle\left\langle\Psi_{2} \mid \Psi_{3}\right\rangle \ldots\left\langle\Psi_{N-1} \mid \Psi_{N}\right\rangle\right\}
\end{aligned}
$$

Eq. (28) can be considered as the general "vertex theorem".

Mukanda and Simon [22] have proved, by developing certain mathematical procedures, that any two normalized vectors in ray space can be connected by a geodesic curve. Therefore one can apply Eq. (28) for the special case in which each of the transitions $\Psi_{r} \rightarrow \Psi_{r+1}$ is a geodesic. But in deriving Eq. (28) we have assumed only that the geometric phase in the "global" transition $\Psi_{r} \rightarrow \Psi_{r+1}$ vanish and this does not necessarily imply that this 
transition is geodesic. However, if we assume that the geometric phase vanish continuously for each of the differential transition $\Psi(s) \rightarrow \Psi(s+\varepsilon)(\varepsilon \rightarrow 0)$ in the interval $s \epsilon\left[s_{1} s_{2}\right]$ then the 'curve' is geodesic and Eq. (28) gets the form [20]:

$$
\begin{aligned}
& \phi_{g}(C)= \\
& -\arg \left\{\left\langle\Psi\left(s_{2}\right) \mid \Psi\left(s_{1}\right)\right\rangle\left\langle\Psi\left(s_{1}\right) \mid \Psi\left(s_{1}+\varepsilon\right)\right\rangle\left\langle\Psi\left(s_{1}+\varepsilon\right) \mid \Psi\left(s_{1}+2 \varepsilon\right)\right\rangle \ldots\right. \\
& \left.\left\langle\Psi\left(s_{1}+(N-1) \varepsilon\right) \mid \Psi\left(s_{2}\right)\right\rangle\right\}
\end{aligned}
$$

where $(N-1) \varepsilon=s_{2}-s_{1}, \quad(N-1) \rightarrow \infty, \quad \varepsilon \rightarrow 0$.

Due to the fact that $\phi_{g}(C)$ is gauge invariant one can use "parallel lift" by which $\langle\Psi(s) \mid \dot{\Psi}(s)\rangle=0$ and then the vanishing of the geometric phase in each of the intervals $(\Psi(s), \Psi(s+\varepsilon))$ implies $\langle\Psi(s) \mid \Psi(s+\varepsilon)\rangle=1$. Then we get the simple expression

$$
\phi_{g}(C)=-\arg \left\langle\Psi\left(s_{2}\right) \mid \Psi\left(s_{1}\right)\right\rangle .
$$

For such cases one may measure $\phi_{g}(C)$ as a relative phase shift in the interference pattern by applying Eq. (13), assuming $|A\rangle=\left|\Psi\left(s_{1}\right)\right\rangle,|B\rangle=\left|\Psi\left(s_{2}\right)\right\rangle$. However, if the development is not given by 'parallel transport' one has to subtract the dynamical phase on the right side of Eq. (30). 


\section{Pancharatnam Phase for Polarized Biphotons}

\section{a. Descriptions of the Biphoton States}

We consider a quantum system formed by two correlated photons - a biphoton, emitted via frequency degenerate colinear spontaneous parametric down conversion (SPDC) [12]. We assume that the biphoton state can be described as

$$
|\Psi\rangle=c_{1}|2,0\rangle+c_{2}|1,1\rangle+c_{3}|0,2\rangle
$$

where $\left(N_{x}, N_{y}\right)$ means a state with $N_{x}$ photons in the horizontal $(x)$ polarization mode and $N_{y}$ photons in the vertical $(y)$ polarization mode, with $N_{x}+N_{y}=2$. The states $|2,0\rangle$ and $|0,2\rangle$ are generated via type-I SPDC and the state $|1,1\rangle$ via type-II SPDC. Arbitrary transformations of the polarization vectors $\left(c_{1}, c_{2}, c_{3}\right)\left[\left|c_{1}\right|^{2}+\left.c_{2}\right|^{2}+\left|c_{3}\right|^{2}=1\right]$ are given by unitary $3 \times 3$ matrix $G$, where $G^{\dagger} G=I$, $\operatorname{det} G=1$, which form a three-dimensional representation of the $\mathrm{SU}(3)$ group.

By passing from the basis $|2,0\rangle,|1,1\rangle,|0,2\rangle$ to the basis

$$
\left|\Psi_{+}\right\rangle=\frac{|2,0\rangle+|0,2\rangle}{\sqrt{2}} ; \quad\left|\Psi_{-}\right\rangle=\frac{|2,0\rangle-|0,2\rangle}{\sqrt{2}} ;\left|\Psi_{0}\right\rangle=|1,1\rangle
$$

one obtains three states that can be transformed into one another by means of only phase-plates. It has been suggested to use polarized biphotons as ternary analogs of two-state quantum systems (qubits) [12,15]. Our aim in 
the present paper is, however, different. We would like to study here possible interference effects, by the use of biphotons which can be related to Pancharatnam and Berry phases in a three-level photonic system. The effect of the loss-free polarization converters (phase-plates) on a biphoton state given by Eq. (31) has been described by Burlakov and Klyshko [14]. For our purpose the effect of the phase-plates is described by a $3 \times 3$ unitary matrix which operates directly on the basis of states given by Eq. (32). This transformation matrix and its properties are described in the next paragraph.

\section{b. Transformations of Biphotons by Phase-Plates}

The effect of polarization converters on a biphoton given by Eq. (31) as $\left(c_{1}, c_{2}, c_{3}\right)$ is described by the transformation matrix [14]:

$$
G=\left(\begin{array}{ccc}
t^{2} & \sqrt{2} t r & r^{2} \\
-\sqrt{2} t r^{*} & |t|^{2}-|r|^{2} & \sqrt{2} t^{*} r \\
r^{* 2} & -\sqrt{2} t^{*} r^{*} & t^{* 2}
\end{array}\right)
$$

Here $t$ and $r$ are amplitude transmission and reflection coefficients of a given converter. We assume a linear phase-plate with optical thickness $\delta$ and orientation $\chi$ relative to the horizontal direction $x$ which corresponds to the transformation $t=\cos \delta+i \sin \delta \cos (2 \chi), r=i \sin \delta \sin (2 \chi)$. For a quarter-wave

plate we have $\delta=\pi / 4$ and then $t=(1+i \cos (2 \chi)) / \sqrt{2}, r=i \sin (2 \chi)) / \sqrt{2}$. A half-wave plate gives $t=i \cos (2 \chi), r=i \sin (2 \chi)$. 
The basis of states $|2,0\rangle,|11\rangle$ and $|0,2\rangle$ can be transformed to the basis of states $\left|\Psi_{+}\right\rangle,\left|\Psi_{-}\right\rangle$and $\left|\Psi_{0}\right\rangle$ as

$$
\left(\begin{array}{l}
\Psi_{+} \\
\Psi_{-} \\
\Psi_{0}
\end{array}\right)=A\left(\begin{array}{c}
2,0 \\
1,1 \\
0,2
\end{array}\right)
$$

where the matrix $\mathrm{A}$ is given by

$$
A=\left(\begin{array}{ccc}
\frac{1}{\sqrt{2}} & 0 & \frac{1}{\sqrt{2}} \\
\frac{1}{\sqrt{2}} & 0 & -\frac{1}{\sqrt{2}} \\
0 & 1 & 0
\end{array}\right)
$$

The normalized biphoton state can be expressed in the new basis by

$$
|\Psi\rangle=d_{1}\left|\Psi_{+}\right\rangle+d_{2}\left|\Psi_{-}\right\rangle+d_{3}\left|\Psi_{0}\right\rangle
$$

where the effect of the polarization converters on the biphoton of Eq. (36) given as $\left(d_{1}, d_{2}, d_{3}\right)$ is described by the transformation matrix:

$$
\begin{aligned}
Q=A G A^{-1}= & \left(\begin{array}{lll}
\frac{t^{2}+r^{2}+r^{* 2}+t^{* 2}}{2} & \frac{t^{2}-r^{2}+r^{* 2}-t^{* 2}}{2} & t r-t^{*} r^{*} \\
\frac{t^{2}+r^{2}-r^{* 2}-t^{* 2}}{2} & \frac{t^{2}-r^{2}-r^{* 2}+t^{* 2}}{2} & t r+t^{*} r^{*} \\
-t r^{*}+t^{*} r & -t r^{*}-t^{*} r & |t|^{2}-|r|^{2}
\end{array}\right)
\end{aligned}
$$

Assuming a linear phase-plate with optical thickness $\delta$ and orientation $\chi$ the matrix $Q$ gets a more explicit form as:

$$
Q=\left(\begin{array}{ccc}
\cos (2 \delta) & i \sin (2 \delta) \cos (2 \chi) & i \sin \delta \sin (2 \chi) \\
i \sin (2 \delta) \cos (2 \chi) & \cos ^{2} \delta-\sin ^{2} \delta \cos (4 \chi) & -\sin (4 \chi) \sin ^{2} \delta \\
i \sin (2 \delta) \sin (2 \chi) & -\sin (4 \chi) \sin ^{2}(\delta) & \cos ^{2} \delta+\sin ^{2} \delta \cos (4 \chi)
\end{array}\right)
$$


An interesting property of the matrix of Eqs. (37) and (38) is that its diagonal matrix elements are real. The relations between the matrix $Q$ operating on the biphoton state $\left(d_{1}, d_{2}, d_{3}\right)$ and Pancharatnam and Berry phases will be the subjects of the following discussions.

\section{c. Biphoton Dynamical and Pancharatnam Phases}

Following the general analysis presented in Section 3 the Pancharatnam phase is developed as a function of the parameter $s$ and this is analogous to the development in time of Berry phase by Schrodinger equation. In order to apply the general theory to phase converters we identify the parameter $s$ as the parameter $\delta$ (or later as $2 \delta$ ) in the transformation matrix $Q$ given by Eq. (38). The input state vector is defined as $\left(d_{1}, d_{2}, d_{3}\right)$ while the output state is obtained by multiplying this input state by the matrix $Q$. We assume that the initial state is developed continuously by changing in the matrix $Q$ the parameter $\delta$ from its initial value zero (giving a unit matrix), to its final value $\delta_{0}$, which corresponds to the optical depth of the converter. By

straightforward calculations we find that the linear phase converter leads to a dynamical phase shift given by:

$$
\begin{aligned}
& \int_{0}^{\delta_{0}} d \delta\left(d_{1}^{*}, d_{2}^{*}, d_{3}^{*}\right) Q^{-1} \dot{Q}\left(\begin{array}{c}
d_{1} \\
d_{2} \\
d_{3}
\end{array}\right)= \\
& \int_{0}^{\delta_{0}} d \delta\left\{2 i \cos (2 \chi)\left[d_{1} d_{2}^{*}+d_{1}^{*} d_{2}\right]+2 i \sin (2 \chi)\left[d_{1} d_{3}^{*}+d_{1}^{*} d_{3}\right]\right\}
\end{aligned}
$$


The result obtained in Eq. (39) is quite simple showing that the integrand in this integral is constant, depending only on the initial state $\left(d_{1}, d_{2}, d_{3}\right)$ and the orientation $\chi$ which has a fixed value for a certain converter. The dynamical phase is obtained by multiplying this integrand by $\delta_{0}$.

'Parallel transport' of the biphoton state vector is obtained by the requirement that the integrand of Eq. (39) vanishes. 'Parallel transport' of the biphoton gives, however, trivial results since it is easy to show that under this condition both the Pancharatnam and geometric phases vanish. This conclusion can be obtained by calculating the imaginary value of $\left\langle\Psi_{\text {in }} \mid \Psi_{\text {out }}\right\rangle$ where $\left|\Psi_{\text {in }}\right\rangle$ is the initial biphoton state vector while $\left|\Psi_{\text {out }}\right\rangle$ is obtained by multiplying this initial state by the transformation matrix $Q$. We get:

$$
\begin{aligned}
& \operatorname{Im}\left\langle\Psi_{\text {in }} \mid \Psi_{\text {out }}\right\rangle=\operatorname{Im}\left\{\left(d_{1}^{*}, d_{2}^{*}, d_{3}^{*}\right) Q\left(\begin{array}{c}
d_{1} \\
d_{2} \\
d_{3}
\end{array}\right)\right\}= \\
& \sin 2 \delta\left\{\cos 2 \chi\left(d_{1}^{*} d_{2}+d_{2}^{*} d_{1}\right)+\sin (2 \chi)\left(d_{1}^{*} d_{3}+d_{1} d_{3}^{*}\right)\right\}
\end{aligned}
$$

Therefore, vanishing of the integrand in Eq. (39), which is the condition for 'parallel transport' implies vanishing also of the Pancharatnam phase. Cases for which the total path is composed by a continuum of differential 'curves' elements, where for each of them the geometric phase vanishes while the geometric phase for the total path might be different from zero can be defined as geodesic curves in its most general meaning. For an infinitesimal evolution of the biphoton state from an initial state $\left(d_{1}, d_{2}, d_{3}\right)$, obtained by using additional thickness $d \delta$, the change in geometric phase vanishes. This conclusion 
can be obtained by using in Eq. (40) the approximation $\sin (2 \delta) \simeq 2 \delta$ and compare the Pancharatnam phase change with that obtained for the dynamical phase change given by Eq. (39). Therefore, the general evolution of the Biphoton state by phase converters can be considered as geodesic curves in a general sense. More basic and restricted definitions of geodesic 'curves' will be given later in the article.

There are other interesting effects which can be observed by linear phase converters:

1) By calculating the eigenvalues and eigenvectors of the transformation matrix $Q$ one can describe cyclic transformations by which the initial state vector is multiplied by $U(1)$ phase factor.

2) Special geodesic developments can be obtained for biphoton states under special conditions, which can lead to interesting interference effects.

3) Under general conditions, the geometric phase can be calculated by using the general expressions of Eq. (18).

\section{d. Eigenvalues and Eigenvectors of the Transformation Matrix}

Since the transformation matrix $Q$ is unitary its eigenvalues are given by complex numbers with a unit absolute value. By calculating the eigenvectors of the matrix $Q$ one finds the initial biphoton states for which the transformation $Q$ produces cyclic transformations i.e., the initial state vec-

tors are multiplied by these complex numbers. The general calculation of 
the eigenvalues of the matrix $Q$ for arbitrary optical depth $\delta$ leads to cubic equations. For simplicity, we demonstrate the general procedure by applying it to relatively simple cases.

For a quarter-wave plate with $2 \delta=\pi / 2$ the determinant for the eigenvalues $\lambda$ is given by

$$
-\lambda^{3}+\lambda^{2}-\lambda+1=0
$$

The roots of Eq. (41) are given by $\lambda= \pm i, 1$ (independent of the orientation $\chi)$. For the eigenvalue $\lambda=i$ the normalized eigenvector is given by

$$
d_{1}=\frac{1}{\sqrt{2}} ; \quad d_{2}=\frac{\cos (2 \chi)}{\sqrt{2}} ; \quad d_{3}=\frac{\sin (2 \chi)}{\sqrt{2}}
$$

Here we have assumed that $d_{1}$ is real and this can be done since the phase of the initial biphoton state is arbitrary. However, the phase factor $e^{i \pi / 2}$ by which the initial state is multiplied for getting the final state vector is well defined and can be observed in interference experiments. The dynamical phase can be calculated for the initial state, given by Eq. (42) with $2 \delta=\pi / 2$, by Eq. (39) as:

$$
\int_{0}^{\pi / 4} d \delta\left\{\cos ^{2}(2 \chi)+2 i \sin ^{2}(2 \chi)\right\}=i \pi / 2
$$

So we find that all the Pancharatnam phase is a dynamical phase. Similar calculations can be given for the roots $\lambda=-i$ and 1 .

For a half-wave plate the straightforward calculations show that the roots are given by $= \pm 1$. This result is obvious since the half-wave plate can be 
considered as the operation of two consecutive quarter-wave plates with the same orientation $\chi$, so that the roots for the half-wave plate are obtained by the squares of the roots of the quarter-wave plates. One finds that the roots for phase-plates with arbitrary optical depth $\delta$ will be given by $e^{ \pm i 2 \delta}, 1$. One can also calculate the eigenvalues and the eigenvectors for multiplication of two (or more) phase plates with different orientations $\chi$. Our interest, however, is to calculate geometric phases for noncyclic developments and for this purpose geodesic developments are described in the next paragraph, for special cases.

\section{e. Geodesic Development of Biphoton States}

Let us assume a phase converter with orientation given by $\cos (2 \chi)=1$. We assume also that this phase converter transform a special initial biphoton state for which $d_{3}=0\left(\left|d_{1}\right|^{2}+\left|d_{2}\right|^{2}=1\right)$. We can easily prove that this biphoton state is developed as a function of $\delta$ along a geodesic 'curve', as the geodesic equations (24) is obeyed. Defining $s=2 \delta$ we obtain

$$
\langle\dot{\Psi}(s) \mid \dot{\Psi}(s)\rangle=\left(d_{1}^{*}, d_{2}^{*}\right)\left(\begin{array}{cc}
-\sin s & -i \cos s \\
-i \cos s & -\sin s
\end{array}\right)\left(\begin{array}{cc}
-\sin s & i \cos s \\
i \cos s & -\sin s
\end{array}\right)\left(\begin{array}{l}
d_{1} \\
d_{2}
\end{array}\right)=1
$$

and as $|\ddot{\Psi}(s)\rangle=-|\Psi(s)\rangle$, equation (24) is fulfilled. Although this biphoton does not obey the 'parallel transport' of Eq. (25) we can use a gauge 
transformation of $|\Psi(s)\rangle$ which will lead to a 'parallel transport'. This gauge transformation can be given according to Eq. (39) by

$$
|\Psi(s)\rangle \rightarrow\left|\Psi^{\prime}(s)\right\rangle=|\Psi(s)\rangle \exp \left\{-i s\left[d_{1} d_{2}^{*}+d_{1}^{*} d_{2}\right]\right\}
$$

The conclusion from this transformation is that for the present biphoton state $|\Psi(s)\rangle$ the geodesic property is preserved although the 'horizontal' property is destroyed. [see the discussions after Eq. (26)]. Since the 'horizontal' property is not preserved one can use Eq. (30) but the dynamical phase should be subtracted from the right side of this equation. One should therefore notice that the geodesic 'curve' described here is basically different from that suggested in Ref. [28].

For the present geodesic 'curve' we find that $\theta=\arg \left\langle\Psi\left(s_{1}\right) \mid \Psi\left(s_{2}\right)\right\rangle$ is given as a function of the optical depth $2 \delta=s$ by

$$
\tan \theta=\tan (s)\left(d_{1}^{*} d_{2}+d_{2}^{*} d_{1}\right)
$$

and the geometric phase is given by

$$
\phi_{g}(C)=\theta-s\left(d_{1}^{*} d_{2}+d_{2}^{*} d_{1}\right)
$$

According to Eq. (46) $\theta=s=2 \delta$ only for the special cases for which $d_{1}^{*} d_{2}+d_{2}^{*} d_{1}=1$, and only under this condition the geometric phase vanishes. For more general cases we find that when $s$ is changing from $\pi / 2-\varepsilon(\varepsilon \rightarrow 0)$ to $\pi / 2+\varepsilon$ there is a jump in the geometric phase of $\pi$. Such phase jumps can be observed [16]. 
In a similar way to the above analysis one can obtain another geodesic 'curve' by using a phase converter with orientation given by $\sin (2 \chi)=1$ and an initial state for which $d_{2}=0\left(\left|d_{1}\right|^{2}+\left|d_{3}\right|^{2}=1\right)$. The present geodesic 'curves' are obtained by the development of only two levels chosen from the three-level system.

In the next paragraph we demonstrate calculations of the geometric phase for a real three-level system. We also explain the difference between the general biphotons transformation which we defined as geodesic 'curves' in the more general sense and the more restricted definition given by Eq. (24).

\section{f. Geometric Phase Obtained by Linear Converters}

By the derivations of Eqs. (39) and (40) we implied that the linear converters produce 'curves' which are geodesic in a general sense. Since the transformation of the biphoton state by the matrix $Q$ of Eq. (38) does not obey the geodesic Eq. (24) we need to justify our assumption also from the mathematical point of view. The geodesic equation (24) is basically a harmonic oscillator equation for the wavefunction $|\Psi(s)\rangle$. We find according to Eqs. (39) and (40) that only the imaginary elements of the matrix $Q$ contribute to Pancharatnam and dynamical phases. The imaginary part of the $Q$ matrix fulfills the equation

$$
\operatorname{Im}(\ddot{Q}+4 Q)=0
$$


which is basically an harmonic oscillator equation explaining the geodesic property of this transformation in its general sense.

Let us demonstrate the use of Eqs. (18) for two consecutive transformations operating on an initial state $\left(d_{1}, d_{2}, d_{3}\right)$, first by using quarter-wave plate with an orientation $\chi=0$ and second by using half-wave plate with orientation $\chi=\pi / 4$. We define the Pancharatnam phase in the first, second and total transformations as $\theta_{P a n}^{(1)}, \theta_{P a n}^{(2)}$, and $\theta_{\text {Pan }}^{(3)}$, respectively. Dynamical phases in the first, second and total transformations are defined as $\theta_{d y n}^{(1)}, \theta_{d y n}^{(2)}$ and $\theta_{d y n}^{(3)}$, respectively. By straightforward calculations we get:

$$
\begin{aligned}
& \tan \theta_{\text {Pan }}^{(1)}=\frac{d_{1}^{*} d_{2}+d_{2}^{*} d_{1}}{\left|d_{1}\right|^{2}+\left|d_{2}\right|^{2}+\left|d_{3}\right|^{2} \sqrt{2}} ; \theta_{\text {Pan }}^{(2)}=\theta_{\text {Pan }}^{(t o t)}=0 ; \\
& \theta_{d y n}^{(2)}=2 \pi\left\{\left(d_{1}^{*} d_{3}+d_{1} d_{3}^{*}\right)+i\left(d_{2} d_{3}^{*}-d_{2}^{*} d_{3}\right)\right\} ; \\
& \theta_{d y n}^{(1)}=\pi\left(d_{1}^{*} d_{2}+d_{2}^{*} d_{1}\right) ; \\
& \theta_{d y n}^{(t o t)}=\theta_{d y n}^{(1)}+\theta_{d y n}^{(2)}
\end{aligned}
$$

We find that the total Pancharatnam phase vanishes and therefore in the present case the total geometric phase is equal to minus the total dynamical phase.

\section{Summary and Discussion}

The present paper has analyzed the transformations of biphoton states which can be obtained by the use of linear phase converters in relation to Pancharatnam and Berry phases. While most of the previous works have treated 
two-level systems we analyze here a special three-level photonic system. In order to understand the present analysis the basic concept of Pancharatnam phase is explained in relation to topological effects. The fundamental properties of Pancharatnam and Berry phases are reviewed for the purpose of using them in the analysis of a specific system.

In Section 2 the relation between Berry phase and topology is explained. The phase obtained by an atomic system developing according to Schrodinger equation can be separated into a dynamical and geometric phase. This topological separation is common for Berry and Pancharatnam phase effects in both atomic and photonic systems. The Berry phase is usually calculated for a closed circuit for which the calculation of phase is gauge invariant. An important development has been made by the use of Pancharatnam phase which gives, after the subtraction of the dynamical phase, a geometric phase which is gauge invariant.

In Section 3 the main properties of the Pancharatnam phase are treated. The basic equation for obtaining the geometric phase in any atomic or photonic system is given by Eq. (18). A special emphasis is made in the present work on geodesic 'curves' which are related to Hilbert space metric. The basic equations for geodesics and for 'parallel transport' are given in Eqs. (24) and (25) respectively. A vertex theorem is developed for 'parallel transport' of a Hilbert state along a geodesic 'curve'. 
The main results of the present work are given in Section 4 for analyzing Pancharatnam phases obtained by the transformations of biphotons using phase-plates. We have used the basis of states $\Psi_{+}, \Psi_{-}$and $\Psi_{0}$ given by Eq. (34) and the general transformation of these states by the phase-plates is given by Eq. (38). A general formula for the dynamical phase obtained by this transformation is given by Eq. (39). In Eq. (40) we have obtained the result for $\operatorname{Im}\left\langle\Psi_{\text {in }} \mid \Psi_{\text {out }}\right\rangle$ and have shown that for an infinitesimal transformation the geometric phase vanishes. We find therefore that the transformation given by the matrix $Q$ of Eq. (38) produces a geodesic curve in its general meaning. Cyclic transformations for the Pancharatnam phases have been obtained by the use of eigenvalues and eigenvectors of the transformation matrix but such transformations give only dynamical phases. Geodesic 'curves' fulfilling the geodesic equation (24) are obtained for special transformations. For such geodesic 'curves' the 'horizontal' property of the 'parallel transport' is destroyed but not the geodesic property. Possible phase jumps of $\pi$ are related to a certain discontinuity in the geometric phase. Although the geodesic equation (24) is fulfilled only for special cases, describing the development of two levels out of the three-level system, it has been shown that the general transformation of the three optical levels by the matrix $Q$ is also geodesic in its general sense. Such geodesic 'curves' are obtained for vanishing geometrical phases for small changes in the transformation matrix, but obtained as a global geometric phase change in the total curve, related to non-additivity of the geometrical phase. This geodesic property has been justified mathematically also by replacing the geodesic equation (24) by the 
present geodesic equation (48). In this new equation only the imaginary part of the $Q$ matrix is taken into account, since the real part of the $Q$ matrix does not contribute to Pancharatnam and geometric phases. The use of the general equation (18) for calculating geometrical phases is demonstrated by a calculation for a special case.

\section{Acknowledgement}

The author would like to thank S.P. Kulik for interesting discussions. 


\section{References}

1. Berry, M.V., 1984, Proc. R. Soc. A. 392, 45-57.

2. Aharonov, Y. and Anandan, J., 1987, Phys. Rev. Lett. 58, 1593-1596.

3. Pancharatnam, S., 1956, Proc. Ind. Acad. Sci. A 44, 247-262.

4. Jordan, T.F., 1988, Phys. Rev. A. 38, 1590-1592.

5. Samuel, J. and Bhandari, R., 1988, Phys. Rev. Lett. 60, 2339-2342.

6. Eguchi, T., Gilkey, P.B. and Hanson, A.G., 1980, Physics Rep. 66, 213-393.

7. Chern, S.S., Chen, W.H. and Lam, K.S., 1998, Lectures on Differential Geometry (Singapore: World Scientific).

8. Kobayashi, S. and Nomizu, K., 1969, Foundations of Differential Geometry (New York: Interscience).

9. Nash, C. and Sen, S., 1983, Topology and Geometry for Physicists (London: Academic Press).

10. Simon, B., 1983, Phys. Rev. Lett. 51, 2167-2170.

11. Ben Aryeh, Y., 2002, J. Mod. Optics, 49, 207-220.

12. Burlakov, A.V., Chekova, M.V., Karabutova, O.A., Klyshko, D.N. and Kulik, S.P., 1999, Phys. Rev. A, 60, R4209-R4212. 
13. Burlakov, A.V., Chekova, M.V., Karabutova, O.A. and Kulik, S.P., 2001, Phys. Rev. A, 64, 041803, 1-4.

14. Burlakov, A.V. and Klyshko, D.N., 1999, JETP Lett. 69, 839-843.

15. Burlakov, A.V., Krivitskiy, L.A., Kulik, S.P., Maslennikov, G.A. and Chekova M.V., July 2002, arxiv:quant-ph/0207096.

16. Bhandari, R., 1997, Phys. Rep., 281, 1-64.

17. Wagh, A.G. and Rakhecha, V.C., 1995, Phys. Lett. A., 197, 107-111.

18. Sjoqvist, E., 2001, Phys. Lett. A. 286, 4-6.

19. Sjoqvist, E., Feb. 2002, arxiv: quant-ph/0202078.

20. Sjoqvist, E., Pati, A.K., Ekert, A., Anandan, J.S., Ericsson, M., Oi, D.K.L. and Vederal, V., 2000, Phys. Rev. Lett., 2845-2849.

21. Ericsson, M., Pati, A.K., Sjoqvist, E., Brannlund, J. and Oi, D.K.L., June 2002, arxiv: quant-ph/0206063.

22. Mukanda, N. and Simon, R., 1993, Annals of Physics, 228, 205-340.

23. Anandan, J. and Aharonov, Y., 1990, Phys. Rev. Lett. 65, 1697-1700.

24. Anandan, J., 1991, Foundations of Physics 21, 1265-1284.

25. Bohm, A., Boya, L.J. and Kendrik, B., 1991, Phys. Rev. A 43, 12061210. 
26. Arvind, Malesh, K.S. and Mukanda, N., 1997, J. Phys. A: Math. Gen. 30, 2417-2431.

27. Sjoqvist, E., 2001, Phys. Rev. A, 63, 035602, 1-4.

28. Sanders, B.C., De Guise, H., Bartlett, S.D. and Zhang, W., 2001, Phys. Rev. Lett. 86, 369-372. 